\title{
MODELOS PROXY, ALTERNATIVA PARA REDUCIR LOS TIEMPOS DE CÓMPUTO DURANTE LA SIMULACIÓN NUMÉRICA
}

Luis C. Monsalve Parra ${ }^{1}$, Jorge M. Padilla Reyes ${ }^{2}$, Samuel F. Muñoz Navarro ${ }^{3}$

*A quien debe dirigirse la correspondencia

\section{RESUMEN}

La simulación numérica de yacimientos es una herramienta que ha venido evolucionando de la mano de la industria petrolera, su objetivo fundamental es predecir el comportamiento de un yacimiento dado y posteriormente encontrar el escenario óptimo para aumentar la recuperación de hidrocarburos. Sin embargo, los estudios de simulación numérica pueden llegar a ser muy costosos por factores como: tamaño de la grilla y la robustez del estudio a realizar (asociado a mayores tiempos de cómputo y de análisis de resultados); además del tiempo que implicaría llegar a evaluar los múltiples escenarios posibles para definir la mejor estrategia de explotación. Es por esta razón, que los ingenieros de yacimientos se apoyan en herramientas estadísticas para aplicar los estudios de simulación numérica orientados al ajuste histórico, análisis de sensibilidad, análisis de incertidumbre, predicción y estrategias de optimización de producción. Dentro de estas herramientas estadísticas se encuentran los Modelos Proxy, los cuales surgen como alternativa para reducir los tiempos de cómputo en estudios de simulación numérica que no requieran un alto grado de exactitud en sus resultados. Este artículo busca contextualizar al lector sobre el significado de los modelos proxy y su utilidad durante los estudios de simulación numérica de yacimientos, además de presentar un ejemplo de aplicación.

Palabras clave: Simulación numérica de yacimientos, Modelos Proxy, Inyección de $\mathrm{CO}_{2}$.

\section{PROXY MODELS, AN ALTERNATIVE TO REDUCE THE COMPUTATIONAL TIME DURING NUMERICAL RESERVOIR SIMULATION}

\begin{abstract}
The reservoir simulation is a tool which had been evolving at the same time than the petroleum industry; its main purpose is to predict the behavior of a reservoir and afterward finding the optimum scenario in order to increase the oil recovery. However, numerical simulation studies could be very expensive because factors such as grid size and detail of the study to develop (It is associated to long computational and analysis times). Furthermore the time it would take to evaluate multiple possible scenarios to define the best exploration strategy. For this reason, field engineers use statistical tools during numerical simulation studies oriented to history match, sensitivity analysis, uncertainty analysis, optimization and forecasting. One of these statistical tools is the Proxy model, it appears as an alternative to reduce computational times which does not need a high accuracy grade in the results. This article looks for a reader contextualization about the meaning of Proxy model and its value during reservoir simulation studies, and also to present an application study.
\end{abstract}

Keywords: Numerical Reservoir Simulation, Proxy Model, $\mathrm{CO}_{2}$ injection.

1. Ingeniero de petróleos. Grupo de Investigación Recobro Mejorado. Universidad Industrial de Santander, UIS. Bucaramanga, Colombia. E-mail: luisc.monsalve@outlook.com

2. Magister en Ingeniería de Hidrocarburos. Grupo de Investigación Recobro Mejorado. Universidad Industrial de Santander, UIS. Bucaramanga, Colombia. E-mail: jorge.padilla1@hotmail.com

3. Magister en Ingeniería de Hidrocarburos. Grupo de Investigación Recobro Mejorado. Universidad Industrial de Santander, UIS. Bucaramanga, Colombia. E-mail: samuel@uis.edu.co 


\section{INTRODUCCIÓN}

El objetivo principal de la ingeniería de yacimientos es el definir la mejor estrategia de explotación de un yacimiento, bajo escenarios de rentabilidad económica. Para evaluar las posibles alternativas de explotación de un yacimiento, los ingenieros se apoyan principalmente en las herramientas software de simulación numérica. La fiabilidad de los resultados obtenidos de la simulación numérica, es dependiente del grado de incertidumbre que exista sobre las propiedades de la roca, fluidos y su distribución en la formación. Por lo tanto, conforme se tenga mayor información del yacimiento evaluado, mayor asertividad tendrán los resultados arrojados por el simulador. Sin embargo, el nivel de robustez del modelo simulación afecta directamente el costo computacional. Siendo el costo computacional la principal limitante en la realización de estudios de simulación numérica, los ingenieros deben apoyarse en herramientas estadísticas para la reducción del número de corridas de simulación planeadas para la evaluación de los posibles escenarios de desarrollo de un yacimiento particular. Una de estas herramientas son los Modelos Proxy, los cuales básicamente permiten predecir el comportamiento de una función objetivo (i.e. producción de fluidos, presión de yacimiento, entre otras) en función de algunos parámetros determinados, a partir, de los resultados de un grupo de simulaciones bases empleadas para la obtención de una función matemática.

Este artículo describe los diferentes métodos empleados para la construcción de modelos Proxy, muestra el procedimiento general para su construcción, y presenta un ejemplo de aplicación de un modelo proxy que permite la evaluación del desempeño (a partir del cálculo del factor de recobro) de un proceso de inyección continua de $\mathrm{CO}_{2}$ miscible, elaborado a partir de datos generados de la simulación numérica del proceso y obtenidos de acuerdo al diseño experimental seleccionado.

\section{MODELOS PROXY}

Los modelos proxy se fundamentan en la integración de métodos estadísticos, como el diseño de experimentos y la metodología de superficie de respuesta (RSM - Response Surface Methodology). El diseño de experimentos permite reducir el número de simulaciones que deben ser realizadas para la obtención de un modelo proxy (Avansi, 2009). Básicamente, el Modelo Proxy hace referencia a una función matemática capaz de reproducir los resultados del simulador (i.e., producción acumulada de aceite, agua o gas, presión del yacimiento, factor de recobro, entre otras), a partir de los resultados arrojados por unas corridas bases y en función de parámetros de entrada que pueden ser propiedades de yacimiento o variables operacionales (i.e., numero de pozos, controles de producción y/o inyección, propiedades petrofísicas, entre otras).

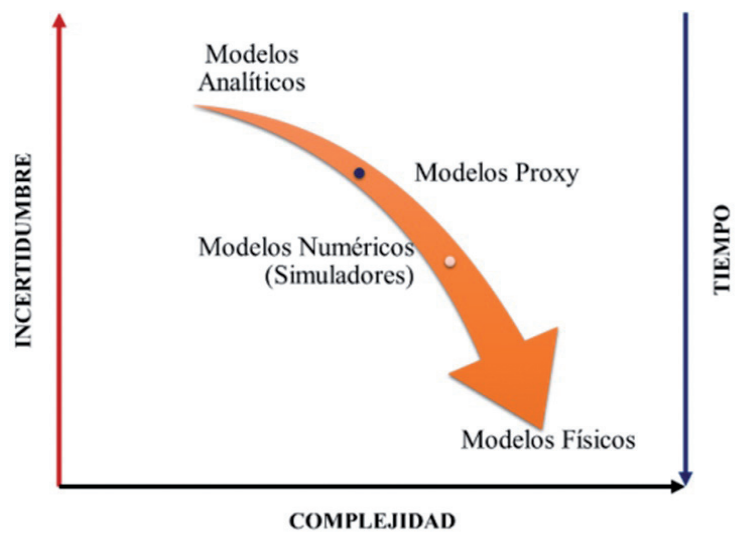

Figura 1. Ubicación de diferentes tipos de modelos empleados durante la simulación numérica. Adaptado de Azad \& Chalaturnyk (2013).

Los modelos Proxy, como se muestra en la figura 1, se asemejan, en cuanto al nivel de complejidad y de incertidumbre, a los modelos analíticos. Estos son una aproximación a un modelo de simulación existente. Estos debe ser capaz de reproducir el comportamiento altamente no lineal del modelo real, pero a su vez debe ser fácil de construir y simple de utilizar (Bevillion \& Mohagerani, 2015).

En general, existen diferentes tipos de modelos proxy, los más empleados en la industria de los hidrocarburos son: Modelos Polinómicos, Modelos tipo Kriging, Modelos de Splines y Redes Neuronales. La calidad de un modelo proxy está dado por su desviación estándar, un valor de 1 indica un ajuste perfecto entre los resultados del proxy y los reales de la simulación, por el contrario un valor de " 0 " corresponde a un mal ajuste. Comúnmente, un modelo proxy con una desviación estándar superior 0.6 es considerado aceptable (Bevillion \& Mohagerani, 2015).

\section{- Modelo Polinómico}

En particular, los modelos provenientes de regresiones polinómicas son los más empleados en la industria petrolera para el análisis y representación de procesos físicos. Esto debido, a su facilidad de entendimiento, flexibilidad y eficiencia computacional (Zubarev, 2009). En general, para formular una regresión de tipo cuadrática se emplea la ecuación 1.

$$
y(x)=\beta_{0} \sum_{i=1}^{n_{d}} \beta_{i} X_{i}+\sum_{j=1}^{n_{d}} \sum_{j=1}^{n_{d}} \beta_{i j} X_{i} X_{j}+\sum_{i=1}^{n_{d}} \beta_{i i} X_{i}^{2}
$$


Donde $x$ es el vector de variables de entrada de longitud $n_{d}, x_{i}$ es un término lineal, $x_{i} x_{j}$ es un término intermedio y $x_{i}^{2}$ es el término cuadrático y $\beta_{0}, \beta_{i}, \beta_{i j}, \beta_{j}$ representan los coeficientes de regresión desconocidos. Los términos beta son determinados a partir de mínimos cuadrados. El uso de un modelo de regresión polinómica incluye la selección de los parámetros a incluir y la estimación de los coeficientes de regresión. Adicionalmente, se debe tener presente que este tipo de modelos no es eficiente para representar fenómenos altamente no lineales en tiempo y espacio.

\section{- $\quad$ Modelos tipo Kriging}

En este modelo se parte del principio que puntos próximos en el espacio tienden a tener valores similares contrario a los puntos más distantes. Es por ello, que basan su análisis en variogramas, por medio de los cuales pueden asignar pesos a los parámetros considerados para la construcción del modelo proxy (Fedutenku, Yang, Card \& Nghiem, 2013). Existen modelos Kriging tipo simple, ordinario y universal, sin embargo el más empleado para la construcción de Proxys es el modelo Kriging ordinario. La ecuación para estimar el valor en el punto de interés es:

$$
\hat{y}(x)=f^{T}(x) \hat{\beta}+r^{T}(x) R^{-1}(Y-F \hat{\beta})
$$

Donde $f(x)$ es el vector que contiene los términos de la función de regresión, $\hat{\beta}$ es el vector de los coeficientes de regresión desconocidos, $r()$ es un vector de correlación y $R$ es una matriz de correlación, $F$ y $Y$ son vectores de valores de la función de regresión para el conjunto de datos de entrada y el vector de respuestas de conjunto de datos de entrada. Para estimar los valores del vector beta se requiere emplear un algoritmo de optimización.

Los modelos proxy construidos a partir de este método reproducen completamente la información empleada para su construcción; sin embargo, podrían no ser muy acertados con combinaciones de parámetros diferentes a los empleados en su construcción. El tiempo empleado para la construcción de este tipo de modelos depende de: el número de variables de entrada, el número de experimentos iniciales (diseño de experimentos), tipo de función y la capacidad computacional.

\section{- $\quad$ Modelo de thin plate splines (TSP)}

Es un esquema de interpolación multidimensional para datos con espaciamientos variables. Se define así:

$$
y(x)=\sum_{i=1}^{n_{d}+1} \beta_{i} m_{i}+\sum_{j=1}^{n_{s}} \theta_{j} U\left(\| x+x^{k} \mid\right)
$$

Donde $m$ es un vector de monomios, $\beta_{i}$ y $\theta_{i}$ representan los coeficientes desconocidos, $U(r)=r^{2} \log (r)$ es un plate spline, $\left\|^{o}\right\|$ es una norma Euclidiana, $x^{k}$ es un punto de muestro por fuera de la data inicial y $x$ es el punto de interés. Los modelos TSP reproducen completamente la data de entrada, sin embargo requieren de un gran número de experimentos para reproducir datos diferentes a los de entrada.

\section{- Redes Neuronales}

Las Redes Neuronales Artificiales, ANN (Artificial Neural Networks) están inspiradas en las redes neuronales bilógicas del cerebro humano. Están constituidas por elementos que se comportan de forma similar a la neurona biológica en sus funciones más comunes. Estos elementos están organizados de forma parecida a la que presenta el cerebro humano.

Las ANN, además de parecerse al cerebro, presentan una serie de características propias del cerebro (i.e. aprenden de la experiencia, generalizan de ejemplos previos a ejemplos nuevos y abstraen las características principales de una serie de datos, Basogain, 2014). En la Figura 2 se presenta un esquema general de una red neuronal. En las ANN, la unidad análoga a la neurona bilógica es llamada elemento procesador, PE o nodo. Un elemento procesador tiene varias entradas y las combina, normalmente con una suma básica. La suma de las entradas es modificada por una función de transferencia y el valor de la salida de esta función de transferencia se pasa directamente a la salida del elemento procesador.

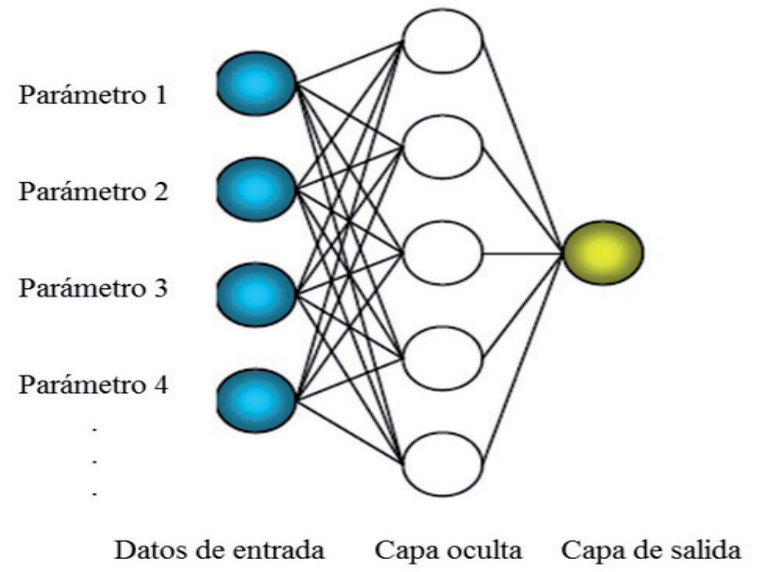

Figura 2. Esquema general de una red neuronal (Zangl, Graf \& Al-kinani, 2006). 
La salida del PE se puede conectar a las entradas de otras neuronas artificiales (PE) mediante conexiones ponderadas correspondientes a la eficacia de la sinapsis de las conexiones neuronales.

Para construir una ANN, es necesario definir una topología: número de capas ocultas, nodos por capa y una función de activación. Esta última, es la encargada de calcular la información de salida para cada nodo. Por otra parte, el número de capas ocultas está limitado por el número de experimentos empleados para construir la ANN. Generalmente, para construir modelos proxy partiendo de redes neuronales se emplea la función de Sigmoid (ecuación 4).

$$
y(x)=\frac{1}{1+e^{-x}}
$$

\section{DISEÑO EXPERIMENTAL Y METODOLOGÍA}

El diseño de experimentos permite obtener la mayor información de la influencia de determinada variable sobre la(s) función(es) objetivo, realizando un mínimo número de corridas. Existen diferentes metodologías para el diseño de experimentos, sin embargo las más usadas para la generación de modelos proxy son: el diseño factorial multinivel y Box-Behnken.

Yaten, Castellini, Guyaguler \& Chen (2005) propusieron un procedimiento general para la construcción de modelos proxy. El primer paso comprende un análisis de sensibilidad de los parámetros basado en un diseño factorial de dos niveles. Este análisis contempla todas las posibles combinaciones entre los valores máximo y mínimo para cada parámetro, el objetivo de este primer paso es el de identificar los parámetros más influyentes en el comportamiento de la función objetivo. El diseño factorial requiere la realización de $2 \mathrm{n}$ simulaciones, donde $\mathrm{n}$ representa el número de variables a evaluar. Sin embargo, Lenchner y Zangl (2005), sugirieron emplear diseños factoriales de tres niveles (mínimo, mejor aproximación, máximo) considerando que la mayoría de los fenómenos encontrados en los yacimientos son altamente no lineales. Luego de realizar el análisis de sensibilidad, se genera un diagrama tornado con la información recolectada y se determinan los parámetros que deben ser empleados para la generación del modelo proxy. Posteriormente, se planean el número total de simulaciones a realizar para la construcción del proxy, para ello se recomienda emplear el método de BoxBehnken, debido a que, el número de experimentos a realizar corresponde a un punto intermedio entre los diseños factoriales de dos y tres niveles. Una vez se cuenta con los resultados de las diferentes simulaciones se procede a generar el proxy, empleando alguno de los modelos antes descritos. Finalmente, se realiza la validación del proxy empleando una combinación de variables que no haya sido considerada para su generación. En caso de que el modelo no sea representativo, se debe realizar un nuevo diseño experimental. En la Figura 3, se presenta un esquema de la metodología.

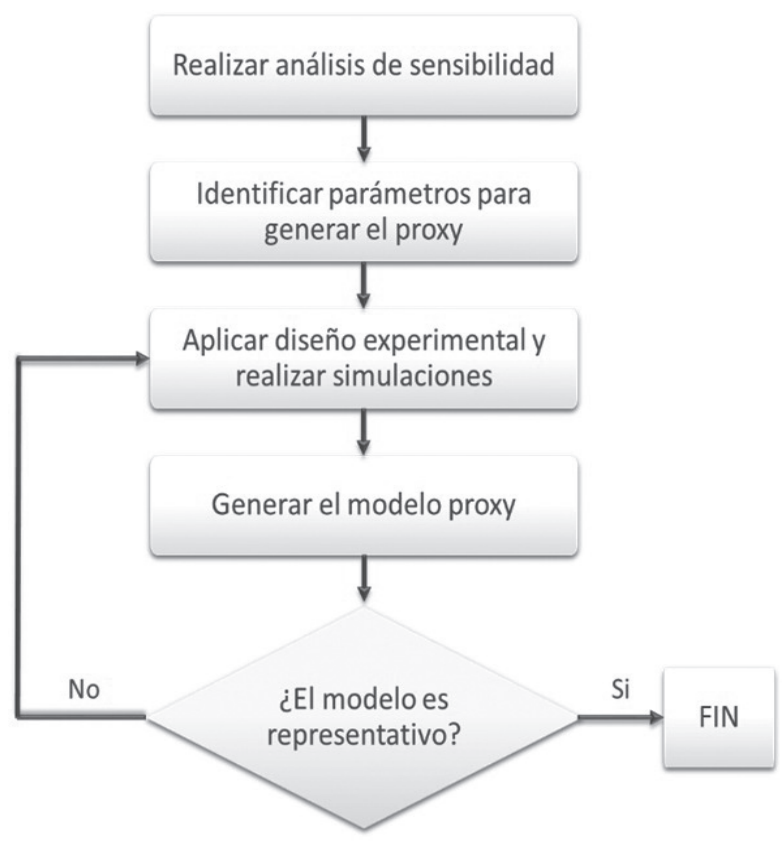

Figura 3. Diagrama de flujo para la construcción de un modelo proxy. (Yaten et al., 2005)

\section{INYECCIÓN DE CO2}

La inyección de $\mathrm{CO}_{2}$ es un proceso de recobro mejorado que consiste en la inyección de dióxido de carbono en estado gaseoso con el fin de reducir la cantidad de aceite remanente atrapado en el yacimiento luego de la aplicación de recobro secundario. La técnica puede llevarse a cabo, dependiendo de la condiciones de presión del yacimiento, como inyección de $\mathrm{CO}_{2}$ miscible o inmiscible; sin embargo, la primera ha demostrado mejores resultados ya que permite la acción de fenómenos y mecanismos de desplazamiento como vaporización, condensación, vaporizacióncondensación, hinchamiento del crudo, reducción de viscosidad, entre otros. Mientras que cuando se aplica bajo condiciones inmiscibles la efectividad del $\mathrm{CO}_{2}$ se limita a la acción del incremento de presión que se logre en las cercanías del pozo inyector. 
La simulación numérica de procesos miscibles como la inyección de $\mathrm{CO}_{2}$, presentan un alto grado de complejidad, para este fin se debe utilizar simuladores composicionales que permitan representar el fenómeno de miscibilidad. El uso de este tipo de simuladores demanda mayores tiempos de cómputo durante la simulación, poder disminuir estos tiempos a través de la utilización de modelos Proxy es una alternativa que aumentaría la eficiencia con la que se lleva a cabo el análisis de estas técnicas.

\section{MODELO DE SIMULACIÓN}

Para simular el proceso de inyección continua de $\mathrm{CO}_{2}$, se empleó un modelo de simulación conceptual. Para definir las propiedades del yacimiento se tomaron como base las características descritas en los criterios de selección propuestos por Shaw \& Bachu (2002), Aladasani \& Bai (2010) y Aladasani y Bai \& Runar (2012). Las características enunciadas por los autores previamente mencionados se resumen en la Tabla 1.

Tabla 1. Criterios de selección para la técnica inyección continua de $\mathrm{CO}_{2}$. (Ayala \& Monsalve, 2014)

\begin{tabular}{|c|c|c|c|}
\hline & $\begin{array}{c}\text { Shaw \& } \\
\text { Bachu }\end{array}$ & $\begin{array}{l}\text { Aladasani } \\
\text { \& Bai }\end{array}$ & $\begin{array}{l}\text { Aladasani, } \\
\text { Bai \& Runar }\end{array}$ \\
\hline & 2002 & 2010 & 2012 \\
\hline Parámetro & \multicolumn{3}{|c|}{ Valor } \\
\hline Gravedad ( $\left.{ }^{\circ} \mathrm{API}\right)$ & 37 & 37 & 35.5 \\
\hline $\begin{array}{l}\text { Sat. Inicial de Aceite } \\
(\%)\end{array}$ & 60 & 46 & 54.43 \\
\hline Temperatura $\left({ }^{\circ} \mathrm{F}\right)$ & 160 & 136.3 & 116.3 \\
\hline Permeabilidad, (mD) & 300 & 201.1 & 20 \\
\hline Porosidad, (\%) & 20 & 14.8 & 11.83 \\
\hline Viscosidad (cP) & - & 2.1 & - \\
\hline Profundidad (pies) & - & 6171.2 & 5780 \\
\hline Buzamiento $\left({ }^{\circ}\right)$ & 20 & - & - \\
\hline Espesor Neto (pies) & 50 & - & - \\
\hline
\end{tabular}

El modelo de simulación fue construido en el módulo GEM/CMG, el grid de simulación cuenta con 9000 celdas, las cuales se distribuyeron con particiones en dirección de cada uno de los ejes de la siguiente forma: 30 en dirección I, 30 en dirección J y 10 en dirección $\mathrm{K}$, la distribución del enmallado de simulación se muestra en la Figura 4.

Teniendo en cuenta las características de un yacimiento candidato a inyección de $\mathrm{CO}_{2}$, se propuso el modelo conceptual de simulación cuyas propiedades se enuncian en la Tabla 2.

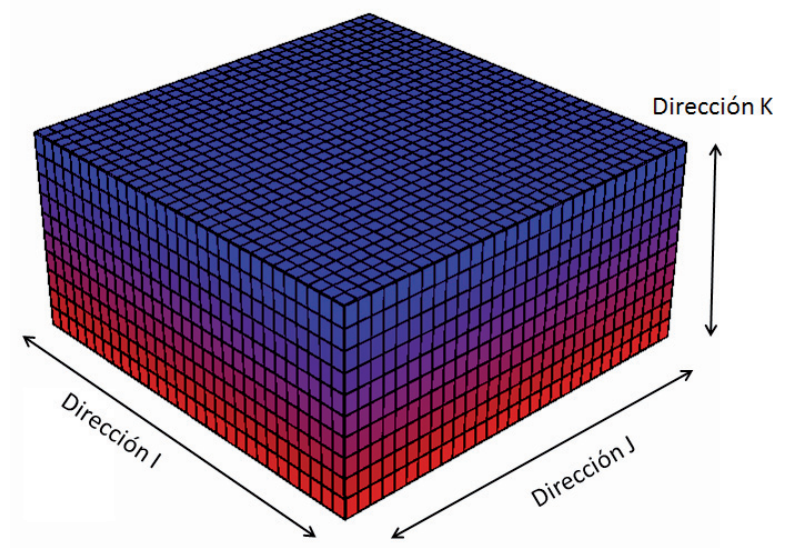

Figura 4. Enmallado del piloto de simulación., CMG, GEM, 2012.

Tabla 2. Características del yacimiento para el modelo conceptual de simulación. (Ayala \& Monsalve, 2014)

\begin{tabular}{|ccc|}
\hline Parámetro & Valor & Unidades \\
\hline Profundidad & 6200 & pies \\
\hline Espesor de la Formación & 40 & pies \\
\hline Porosidad & 15 & $\%$ \\
\hline Permeabilidad I & 15 & $\mathrm{mD}$ \\
\hline Permeabilidad J & 15 & $\mathrm{mD}$ \\
\hline Permeabilidad K & 3 ó & $\mathrm{mD}$ \\
\hline Saturación de Agua Inicial & $\left(0.2^{*} \mathrm{Ki}\right)$ & $\%$ \\
\hline Compresibilidad de la Roca & 30 & $1 / \mathrm{psi}$ \\
\hline Presión Inicial & 2700 & $\mathrm{Psi}$ \\
\hline Temperatura de Yacimiento & 186 & ${ }^{\circ} \mathbf{F}$ \\
\hline
\end{tabular}

El modelo de fluidos fue construido en el módulo WINPROP/CMG, tomando información PVT de un crudo liviano. En la Tabla 3 se presenta la composición del fluido de interés.

Tabla 3. Distribución de los pseudo-componentes del fluido en el modelo conceptual. (CMG Miscible Tutorial, 2012)

\begin{tabular}{ccc} 
Número & Pseudo-componentes & Fracción Molar \\
\hline 1 & $\mathrm{CO}_{2}$ & 0,0128 \\
\hline 2 & $\mathrm{~N}_{2} \mathrm{a} \mathrm{C} 1$ & 0,1275 \\
3 & $\mathrm{C}_{2} \mathrm{a} \mathrm{nC}_{4}$ & 0,2069 \\
\hline 4 & $\mathrm{iC}_{5} \mathrm{a} \mathrm{C}_{7}$ & 0,1712 \\
\hline 5 & $\mathrm{C}_{8} \mathrm{a} \mathrm{C}_{12}$ & 0,1766 \\
\hline 6 & $\mathrm{C}_{13} \mathrm{a} \mathrm{C}_{20}$ & 0,1265 \\
\hline 7 & $\mathrm{C}_{21} \mathrm{a} \mathrm{C}_{30}$ & 0,1785 \\
\hline
\end{tabular}

Finalmente, para completar la definición del modelo conceptual de simulación y teniendo en cuenta que se modela un proceso miscible se debe incluir la siguiente información: 
- Curvas de permeabilidad.

- Pruebas de presión volumen y temperatura.

- Composición del solvente.

- Interacción fluido-solvente

${ }^{\circ}$ Prueba de hinchamiento.

${ }^{\circ}$ Prueba de miscibilidad.

La información antes mencionada para la elaboración del modelo de fluidos fue tomada del trabajo de CMG Miscible Tutorial (2012).

Para representar la inyección continua de $\mathrm{CO}_{2}$ se utilizó un patrón de cinco puntos invertido tal como se muestra en la Figura 5, el cual consta de cuatro pozos productores $\mathrm{y}$ un pozo inyector en el centro del patrón.

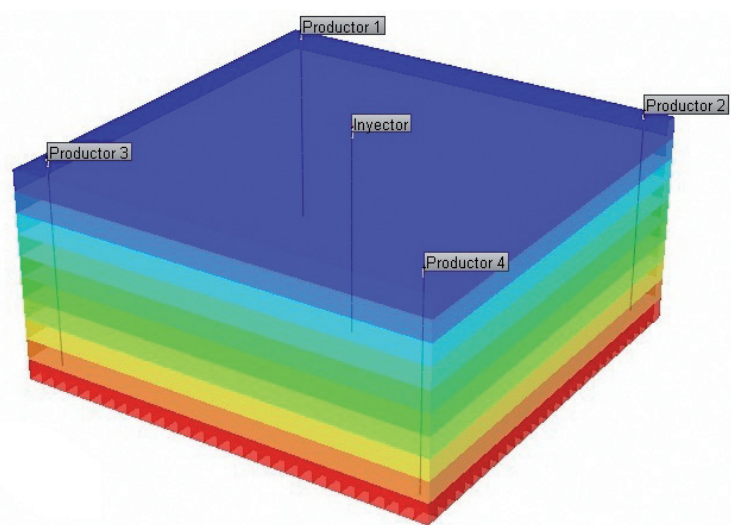

Figura 5. Arreglo de pozos dentro del modelo de simulación, CMG, BUILDER, 2012.

\section{ANÁLISIS DE SENSIBILIDAD Y SELECCIÓN DE VARIABLES}

Para la elaboración del proceso proxy, se siguió la metodología presentada en la Figura 3. El primer paso consiste en la aplicación de un análisis de sensibilidad para la determinación de las variables representativas del proceso. Este análisis se llevó a con el módulo CMOST/CMG, siguiendo la metodología descrita a continuación:

1. Selección del caso base: Se partió de la aplicación de la técnica bajo condiciones base de Presión inicial, permeabilidades, espesor y tasa de inyección de gas $\left(\mathrm{CO}_{2}\right)$.

2. Selección de los parámetros a sensibilizar: Los parámetros seleccionados para el caso de estudio fueron: Presión inicial de yacimiento [REPRES], Permeabilidad vertical [PERMVER] y horizontal [PERMHOR], Espesor productor [THICK], Presión máxima de inyección [BHPMAX] y Tasa máxima de inyección del gas [STGMAX]. Esta selección se basó en la relevancia de los mismos expresada en la literatura.

3. Selección de la función objetivo: La función objetivo sobre la cual se estudiará el efecto de las variables sobre los resultados es el Factor de Recobro.

4. Determinación de los valores críticos (máximos y mínimos) de las variables independientes: La tabla 4 muestra en detalle los valores entre los cuales oscilaron las variables para el estudio de su efecto.

5. Análisis de la incidencia de las variables independientes sobre los resultados de las funciones objetivo: La aplicación de la metodología descrita; permitió definir objetivamente qué variables son las que tienen más incidencia sobre los resultados y/o desempeño de la técnica de recobro en estudio. La Figura 6 muestra la incidencia porcentual de cada variable sobre el desempeño de la técnica en estudio.

Tabla 4. Valor máximo y mínimo de las variables independientes. (Ayala \& Monsalve, 2014)

\begin{tabular}{|c|c|c|c|}
\hline Variable & Valor base & $\begin{array}{l}\text { Valor } \\
\text { mínimo }\end{array}$ & $\begin{array}{l}\text { Valor } \\
\text { máximo }\end{array}$ \\
\hline REPRES (psi) & 2.700 & 2.000 & 4.000 \\
\hline PERMHOR (mD) & 15 & 5 & 100 \\
\hline PERMVER (mD) & $\begin{array}{c}0,2 * \\
\text { PERMHOR }\end{array}$ & $\begin{array}{c}0,1 * \\
\text { PERMHOR }\end{array}$ & $\begin{array}{c}0,5 * \\
\text { PERMHOR }\end{array}$ \\
\hline THICK (pies) & 40 & 10 & 100 \\
\hline BHPMAX (psi) & 4.000 & 3.000 & 5.000 \\
\hline $\begin{array}{l}\text { STGMAX }\left(\text { pies }^{3 /}\right. \\
\text { día) }\end{array}$ & $2^{\prime} 000.000$ & $1^{\prime} 000.000$ & 3'000.000 \\
\hline
\end{tabular}

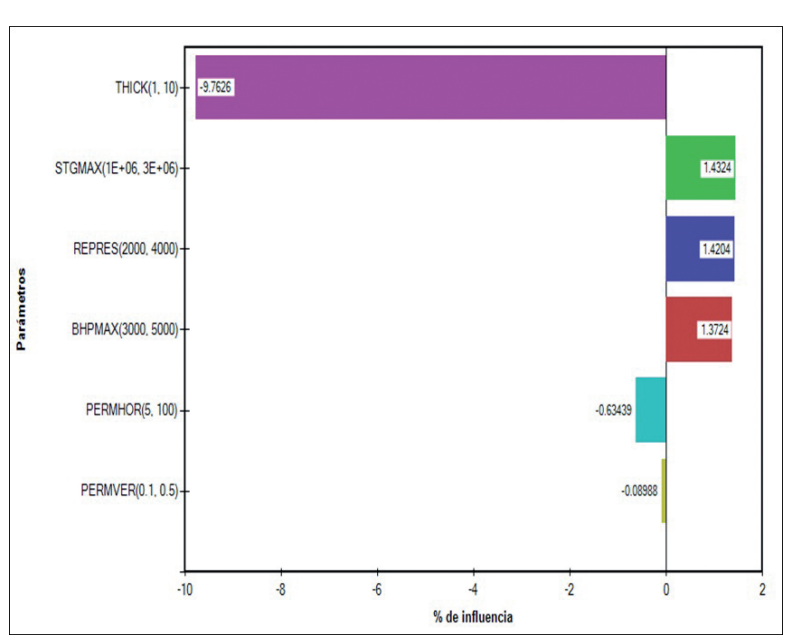

Figura 6. Diagrama tornado sobre la incidencia de las variables sobre el factor de recobro. (Ayala \& Monsalve, 2014) 


\section{CONSTRUCCIÓN Y VALIDACIÓN DEL MODELO PROXY}

Teniendo en cuenta los resultados observados del análisis de sensibilidad, se puede concluir que los parámetros más incidentes en sobre el comportamiento del factor de recobro durante un proceso de inyección de $\mathrm{CO}_{2}$ son: el espesor de la formación de interés [THICK], la Presión inicial del yacimiento [REPRES] y la tasa de inyección del gas [STGMAX].

La razón por la cual el proceso se ve afectado fuertemente por el espesor; es que al tener mayores valores de esta variable se hará más difícil el cubrimiento de la zona de interés por el gas inyectado, disminuyéndose así su efectividad y el grado de miscibilidad que puede alcanzar de manera uniforme en el yacimiento.

La importancia de la presión inicial de yacimiento está dada por su estrecha relación con la miscibilidad del gas inyectado con el fluido de interés, mientras mayor sea la presión inicial de yacimiento se asegura una mejor probabilidad de que el proceso de recobro con gas sea miscible, mientras que al ser menor este parámetro, difícilmente se alcanza la Presión Mínima de Miscibilidad (PMM), la cual corresponde a la presión mínima a la cual se presentará miscibilidad entre el solvente inyectado $\left(\mathrm{CO}_{2}\right.$ en este caso) y el fluido de interés (Hidrocarburo), produciendo un desplazamiento inmiscible, este último con resultados no muy buenos desde el punto de vista de factor de recobro alcanzado al finalizar el proyecto.

Una vez definidas las variables a utilizar para la creación del modelo Proxy, se debe hacer el diseño de experimentos, el cual define, de acuerdo al tipo de diseño, cuántos experimentos se deben realizar con el fin de obtener una muestra representativa de datos $\mathrm{y}$ resultados que representen el comportamiento de la variable en estudio del proceso.

Para el modelo Proxy desarrollado en esta investigación se utilizó un diseño de experimentos tipo Box-Behnken para el cual, teniendo en cuenta que se seleccionaron 3 variables, que representa 15 corridas (experimentos), la elaboración del diseño experimental tipo Box Behnken se detalla en el libro "Diseño y análisis de experimentos", Montgomery, 2002, este corresponde al diseño experimental recomendado el cual demanda 12 corridas menos que si se utilizara el diseño factorial de 3 niveles. En la Tabla 5 se muestran los valores mínimo, intermedio y máximo asignados para las variables según el diseño experimental.
Tabla 5. Valores mínimo, intermedio y máximo de las variables asignadas.

\begin{tabular}{cccc|} 
Valor & $\begin{array}{c}\text { Espesor } \\
\text { (pies) }\end{array}$ & $\begin{array}{c}\text { Tasa de } \\
\text { inyección }\left(\mathrm{pies}^{3} /\right. \\
\text { día) }\end{array}$ & $\begin{array}{c}\text { Presión inicial } \\
\text { (psi) }\end{array}$ \\
\hline Bajo & 30 & $1^{\prime} 000.000$ & 2.000 \\
\hline Intermedio & 40 & $2^{\prime} 000.000$ & 2.300 \\
\hline Alto & 50 & $3^{\prime} 000.000$ & 2.700 \\
\hline
\end{tabular}

En la Tabla 6 se muestra diseño experimental empleado para la generación de los datos que fundamentan la construcción del Modelo Proxy.

Tabla 6. Diseño experimental Box-Behnken para la generación de los datos.

\begin{tabular}{cccc|}
$\begin{array}{c}\text { Experimento } \\
\text { (Corrida de } \\
\text { simulación) }\end{array}$ & $\begin{array}{c}\text { Tasa de } \\
\text { inyección } \\
\left(\text { MPCD }^{1}\right)\end{array}$ & $\begin{array}{c}\text { Presión de } \\
\text { yacimiento (Psi) }\end{array}$ & $\begin{array}{c}\text { Espesor } \\
\text { (Pies) }\end{array}$ \\
\hline 1 & 2 & 2.000 & 40 \\
\hline 2 & 3 & 2.700 & 30 \\
\hline 3 & 2 & 3.200 & 30 \\
\hline 4 & 1 & 3.200 & 40 \\
\hline 5 & 1 & 2.000 & 40 \\
\hline 6 & 1 & 2.700 & 30 \\
\hline 7 & 2 & 2.700 & 50 \\
\hline 8 & 2 & 2.000 & 40 \\
\hline 9 & 2 & 3.200 & 50 \\
\hline 10 & 2 & 2.700 & 40 \\
\hline 11 & 2 & 2.700 & 40 \\
\hline 12 & 3 & 3.200 & 40 \\
\hline 13 & 2 & 2.700 & 40 \\
\hline 14 & 2 & 2.000 & 30 \\
\hline 15 & 1 & 2.700 & 50 \\
\hline
\end{tabular}

Teniendo en cuenta los resultados del factor de recobro generados al simular cada uno de los experimentos (corridas de simulación) diseñados en la Tabla 6, se tiene la información necesaria para la construcción del modelo Proxy, el cual corresponde a un Modelo Polinómico, expresado en la ecuación (5).

$$
\begin{aligned}
F R=5,5628414 * q_{\mathrm{Co}_{2}}+0,00535549 * P_{i} \\
-0,35797395 * h+35,7766316
\end{aligned}
$$

Los resultados que se obtiene con el Modelo Proxy se comparan con los resultados obtenidos de la simulación en la Tabla 7, lo que evidencia un buena aproximación, teniendo en cuenta que una corrida de simulación del modelo de simulación a partir del cual se generaron los datos puede tardar más de 2 horas, el modelo Proxy ofrece una alternativa para obtener respuestas inmediatas y tener una visión de bajo qué parámetros se puede llevar a cabo el proceso, que generen resultados positivos que hagan viable la técnica.

1. MPCD: Millones de pies cúbicos por día. 
Tabla 7. Comparación del Factor de Recobro obtenido con la simulación y con el Modelo Proxy

\begin{tabular}{cccc}
\multicolumn{4}{c}{ Resultados Factor de Recobro (\%) } \\
$\begin{array}{c}\text { Experimento } \\
\text { (Corrida de } \\
\text { simulación) }\end{array}$ & Experimental & Modelo Proxy & $\begin{array}{c}\text { Error } \\
(\%)\end{array}$ \\
\hline 1 & 43,351 & 43,294 & 0,131 \\
\hline 2 & 56,270 & 56,186 & 0,150 \\
\hline 3 & 56,498 & 53,301 & 5,659 \\
\hline 4 & 42,421 & 44,158 & 4,095 \\
\hline 5 & 38,212 & 37,731 & 1,259 \\
\hline 6 & 43,581 & 45,060 & 3,393 \\
\hline 7 & 43,133 & 43,463 & 0,765 \\
\hline 8 & 43,351 & 43,294 & 0,131 \\
\hline 9 & 47,612 & 46,141 & 3,090 \\
\hline 10 & 47,087 & 47,043 & 0,094 \\
\hline 11 & 47,087 & 47,043 & 0,094 \\
\hline 12 & 52,980 & 55,284 & 4,348 \\
\hline 13 & 47,087 & 47,043 & 0,094 \\
\hline 14 & 46,728 & 46,874 & 0,313 \\
\hline 15 & 38,416 & 37,901 & 1,342 \\
\hline
\end{tabular}

Luego de la generación del modelo, el paso final corresponden a la validación del mismo, donde se observa el comportamiento factor de recobro, con el establecimiento previo del margen de error permitido (Error $<10 \%$ ), bajo parámetros que están dentro del rango de aplicación pero que no se tuvieron en cuenta como experimentos del diseño experimental. En la Tabla 8 se muestran los valores utilizados para la validación del modelo y la Tabla 9 los resultados obtenidos que lo validan

Tabla 8. Valores utilizados para la validación del modelo.

\begin{tabular}{cccc}
$\begin{array}{c}\text { Experimento } \\
\text { (Corrida de simu- } \\
\text { lación) }\end{array}$ & $\begin{array}{c}\text { Tasa de } \\
\text { inyección } \\
\text { (MPCD) }\end{array}$ & $\begin{array}{c}\text { Presión de yaci- } \\
\text { miento (Psi) }\end{array}$ & $\begin{array}{c}\text { Espesor } \\
\text { (Pies) }\end{array}$ \\
\hline 16 & 2 & 2.000 & 35 \\
17 & 3 & 2.300 & 30 \\
18 & 1,5 & 3.200 & 30 \\
\hline 19 & 2,5 & 2.800 & 45 \\
\hline
\end{tabular}

Tabla 9. Resultados validación modelo Proxy.

\begin{tabular}{|cccc|}
\multicolumn{4}{c}{ Resultados Factor de Recobro (\%) } \\
$\begin{array}{c}\text { Experimento } \\
\text { (Corrida de } \\
\text { simulación) }\end{array}$ & $\begin{array}{c}\text { Resultado } \\
\text { Experimental }\end{array}$ & $\begin{array}{c}\text { Modelo } \\
\text { Proxy }\end{array}$ & Error (\%) \\
\hline 16 & 45,594 & 45,084 & 1,119 \\
\hline 17 & 52,079 & 54,044 & 3,773 \\
\hline 18 & 54,083 & 50,519 & 6,590 \\
\hline 19 & 51,333 & 48,570 & 5,383 \\
\hline
\end{tabular}

\section{CONCLUSIONES}

El principal aporte de este documento, fue resaltar la utilidad de los modelos proxy como alternativa para la reducción de los tiempos de cómputo, asociados a los estudios de simulación numérica de yacimientos. Sin embargo, es necesario aclarar que los modelos proxy son recomendados para estudios, en los cuales se requieren resultados rápidos sin un alto grado de exactitud.

En general, los modelos proxy construidos a partir de regresiones polinómicas son, de acuerdo a la literatura, los más utilizados en la industria, debido a que son más sencillos de generar y tienen un nivel de asertividad aceptable.

Aunque la diferencia entre haber realizado la totalidad de simulaciones posibles para el caso de estudio presentado y las requeridas para obtener un modelo proxy representativo se reduce a unas cuantas horas. La verdadera utilidad de los modelos proxy, se ve reflejada cuando la simulación numérica se realiza con modelos robustos a escala de campo y con un mayor número de variables, donde la diferencia en los tiempos de cómputo es de varios días.

\section{AGRADECIMIENTOS}

Los autores agradecen a la Universidad Industrial de Santander y al Grupo de Investigación Recobro Mejorado por el apoyo recibido.

\section{REFERENCIAS}

1. ALADASANI, A. y BAI, B. Recent Developments and Update Screening Criteria of Enhanced Oil Recovery Techniques. En SPE 130726 presentado en CPS/SPE International Oil \& Gas Conference and Exhibition, Beijing, China, 2010.

2. ALADASANI, A., BAOJUN, B., NYGAARD, R. A Selection Criterion for $\mathrm{CO} 2$ Enhanced Oil Recovery and Dispersion Modeling of High - Pressure $\mathrm{CO}_{2}$ Release. En SPE 152998 presentado en SPE Western Regional Meeting, California, USA, 2012

3. AVANSI, G. Use of Proxy Models in the Selection of Production Strategy and Economic Evaluation of Petroleum Fields. En SPE 129512 presentado en SPE Annual Technical Conference and Exhibition, New Orleans, USA, 2009.

4. AYALA, A. y MONSALVE, L. Evaluación técnica de un proceso de inyección continúa de $\mathrm{CO}_{2}$ miscible 
mediante simulación numérica. Universidad Industrial de Santander, UIS. Bucaramanga 2014. $1-130 \mathrm{p}$.

5. AZAD, A. y CHALATURNYK, R. J. Application of Analytical Proxy Models in Reservoir Estimation for SAGD Process: UTF-Project Case Study. En SPE 165576, publicado en Journal of Canadian Petroleum Technology, 2013.

6. BASOGAIN, X. Curso de Redes Neuronales Artificiales y sus Aplicaciones: documento electronico tomado de internet. [Fecha de consulta 5 de febrero de 2016], disponible en: <http://www. frro.utn.edu.ar/repositorio/catedras/quimica/5_anio/ orientadora1/monograias/matich-redesneuronales. pdf $>$

7. BEVILLION, D. y MOHAGERANI, S. A Miscible EOR Project in a Mature, Offshore, Carbonate Middle East Reservoir - Uncertainty Analysis With Proxy Models Based on Experimental Design of Reservoir Simulations. En SPE 175642 presentado en SPE Reservoir Characterisation and Simulation Conference and Exhibition, Abu Dhabi, UAE, 2015.

8. COMPUTER MODELLING GROUP LTD. $\mathrm{CO}_{2}$ EOR Miscible Tutorial Using WinProp. 2012.

9. FEDUTENKO, E., YANG, C., CARD, C., NGHIEM, L.X. Time-Dependent Proxy Modeling of SAGD Process. En SPE 165395 presentado en
SPE Heavy Oil Conference Canadá, Calgary, 2013.

10. LECHNER, J. P., ZANGL, G. Treating Uncertainties in Reservoir Prediction with Neural Networks. En SPE 94357 presentado en SPE Europec/EAGE Annual Conference, Madrid, España, 2005.

11. MONTGOMERY, Douglas C. Diseño y Análisis de Experimentos. Grupo Editorial Iberoamerica. 2002.

12. SHAW, J. y BACHU, S. Screening, Evaluation, and Ranking of Oil Reservoirs Suitable for $\mathrm{CO}_{2}-$ Flood EOR and Carbon Dioxide Sequestration. En PETSOC Journal Paper. 2012.

13. YATEN, B., CASTELLINI, A., GUYAGULER, B., CHEN, W.H. A Comparison Study on Experimental Design and Response Surface Methodologies. En SPE 93347 presentado en SPE Reservoir Simulation Symposium, Houston, USA, 2005.

14. ZANGL, G., GRAF, T., AL-KINANI, A. Proxy Modeling in Production Optimization. En SPE 100131 presentado en SPE Europec/EAGE Annual Conference, Viena, Austria, 2006.

15.ZUBAREV, D.I. Pros and Cons of Applying Proxy-Models as a Substitute for Full Reservoir Simulations. En SPE 124815 presentado en SPE Annual Technical Conference and Exhibition held in New Orleans, USA, 2009.

Recepción: 2 de julio de 2016

Aceptación: 8 de octubre de 2016 